\title{
Nacionalización de las memorias colectivas y reproducción de riesgos en regiones fronterizas latinoamericanas/
}

\author{
Collective Memory Nationalization and Risk Reproduction \\ in Latin-American Border Regions
}

\author{
Rogelio Altez
}

Universidad Central de Venezuela

Los procesos nacionales fracturan las memorias ancestrales anclando códigos y referentes a sus límites soberanos y arbitrarios. Con ello, los riesgos asociados a fenómenos naturales son comprendidos fragmentariamente en regiones fronterizas. Proponemos utilizar herramientas históricas para reducir los riesgos en esas regiones y reconstruir memorias colectivas desde la investigación documental con ese fin.

Palabras Clave: Memoria colectiva; Riesgos; Desastres; Fronteras; Latinoamérica.

Nationals process fragmenting ancestral memory by anchoring codes and referents at the arbitrary limits of national sovereignty. Thus, risks associated to natural hazards are understood in fragmentary way, especially in border regions. We propose to use historical tools to reduce risks in those regions and reconstruct collective memory through documentary research for this goal.

KeYwords: Collective memory; Risks; Disasters; Borders; Latin America. 


\section{Fronteras en las memorias}

Los desastres parecen no tener un espacio reservado en la memoria de las historiografías tradicionales. Embebidas en una persecución interminable que va tras los pasos de los héroes, de los cambios institucionales y de las decisiones políticas, las adversidades especialmente asociadas a fenómenos naturales apenas colorean las aventuras de los protagonistas de siempre. Esto es así, y con gran énfasis, en el caso de las «Historias Patrias» latinoamericanas, ${ }^{1}$ construidas al filo de su necesidad imperante de instituir un relato que justificara sus surgimientos y darle sentido a la nación recién fundada. Al único desastre al que estos relatos prestan atención es a la guerra, entendida como el escenario que pone a prueba el temple de sus personajes y el pulso de sus plumas. ${ }^{2}$

En la lógica interpretativa de esa historia tradicional, los fenómenos potencialmente destructores o conducentes a padecimientos extremos, como los terremotos, las lluvias, las inundaciones, los aludes, las sequías, las erupciones, los huracanes, o las anomalías meteorológicas de gran extensión, todos ellos de regular comportamiento, parecen pertenecer a la «otra historia», la de la naturaleza, aquella que no construye héroes. Rafael María Baralt y Ramón Díaz, conspicuos representantes de la Historia Patria venezolana, habrían sentenciado en 1841 que «Los trabajos de la paz no dan materia a la historia: cesa el interés que ésta inspira cuando no puede referir grandes crímenes, sangrientas batallas, o calamitosos sucesos». ${ }^{3}$ No obstante, esas calamidades a las que se refieren se hallan vinculadas, siempre, a las andanzas de los héroes, y no necesariamente a las adversidades características asociadas con el comportamiento de la naturaleza.

Resulta todo un enigma, además, el hecho de que estos fenómenos infundan temor generalizado y al mismo tiempo no ocupen un lugar distinguido entre los objetos de estudio historiográficos o sociológicos. Es esta una relación contradictoria, pero es, asimismo, el producto de una forma de historiar y de una forma de olvidar. En ese sentido, esos fenómenos naturales conducentes a padecimientos extremos parecen hallarse condenados a formas de interpretación en las que su condición de amenazas se potencia y exacerba, mientras que las vulnerabilidades con las que se articulan parecen profundizarse con el paso del tiempo, puesto que ese

1 Sobre Historias Patrias, véase el trabajo de Carrera Damas, 1979.

2 Altez, 2012, 108.

3 Baralt y Díaz, 1939, II, 144. 
olvido con el que se representan en la memoria colectiva, les hace crecer como amenaza en relación directamente proporcional con la vulnerabilidad que les padece.

Nos proponemos explorar en este trabajo ese efecto de olvido producido por la construcción de las memorias colectivas nacionales, especialmente en Latinoamérica, a partir de las cuales se ha generado al relato de la nación, así como a los referentes más representativos de esa relación subjetiva con el pasado y los procesos históricos y sociales que le son propios a las comunidades que habitan, especialmente, en las regiones fronterizas de estos países.

Pensamos que en las fronteras nacionales, cuyo origen pertenece al mismo contexto histórico en casi todos los casos latinoamericanos, esas comunidades que han existido asentadas en dichas regiones desde antes de los decretos fundadores de las nuevas naciones, han padecido la fragmentación de sus memorias colectivas en favor del relato de la nación, y los procesos subjetivos construidos a partir de sus relaciones concretas y simbólicas con los medioambientes en los que se hallan enclavadas, han sufrido la intervención de las estrategias socializadoras de los países que hoy dividen su entorno entre soberanías y jurisdicciones que fracturan la continuidad temporal de esa relación.

Esto, desde luego, afecta directamente a la percepción y producción de los riesgos y las vulnerabilidades. Más aún cuando las políticas institucionales que han atendido (exitosa o parcialmente) al problema de las amenazas naturales en esas regiones, solo alcanzan a hacerlo desde una de las caras de esas fronteras, contribuyendo a la fragmentación de esas mismas políticas y a la fractura de sus resultados. Los fenómenos naturales que despliegan sus regularidades en esos entornos nada saben de jurisdicciones o soberanías, y sus efectos se van transformando históricamente al ritmo con el que las relaciones de poder y los intereses de turno intervienen sobre la relación que las comunidades desarrollan con sus comportamientos. Tal transformación parece significarse en el aumento de los efectos adversos asociados a sus manifestaciones regulares, figurando que los fenómenos se antojan más catastróficos con el paso del tiempo. No es el fenómeno el que cambia, sino la sociedad que convive con sus manifestaciones.

Creemos que si esa memoria, hoy fragmentada e intervenida en favor del relato nacional, se recupera trascendiendo los límites que separan a esos países y se devuelve a esa relación concreta y simbólica que allí se produce y reproduce socialmente, probablemente se contribuya a la construcción 
de herramientas subjetivas y efectivas capaces de enfrentar con mejores respuestas al retorno de los fenómenos potencialmente destructores que allí tienen lugar. Esto no es un método, sino una reflexión surgida a partir de la interpretación del hecho indefectible que se sucede a la producción de las memorias colectivas de la nación: el del olvido igualmente colectivo.

\section{Sobre la nacionalización de las memorias colectivas}

En ciencias sociales hablar de memoria es hacer referencia a la memoria colectiva, siempre. Toda consideración sobre la memoria en el estudio de las sociedades o de las culturas hace alusión a mucho más que el individuo, de manera que aquello que torna en referente común a un hecho del pasado trasciende la experiencia individual del recuerdo y se asienta sobre aspectos que superan el tiempo existencial, desplazándose en velocidades estructurales, imperceptibles a escala individual. ${ }^{4} \mathrm{La}$ memoria, en este sentido, es colectiva porque logra extenderse más allá de una vida y no depende de las vicisitudes de un sujeto, sino de lo que sostiene simbólicamente a una sociedad en su cohesión relacional a través del tiempo. De allí que, en tanto que colectiva, necesita de vehículos capaces de transportar sus referentes por encima de la voluntad personal, con el objeto de prolongar y consolidar su eficacia. Por ello no se trata de un ente, de un espíritu transcendental o una naturaleza propia de las sociedades: la memoria solo puede sostenerse y ser eficaz si se articula simbólicamente con las relaciones de poder, el único aspecto del entramado social y cultural que produce, reproduce o transforma las estructuras profundas. ${ }^{5}$ En el caso de los Estados nación, esa articulación es una responsabilidad formal, pública y oficial, y las naciones latinoamericanas representan un transparente ejemplo al respecto. ${ }^{6}$

Estas naciones se fundaron sobre los efectos concretos e ideológicos de las independencias. Fue a partir de allí que se «creó» a la nación, y en ese proceso se levantaron los referentes de su memoria colectiva. No

4 Hacemos alusión aquí a la noción antropológica de cambio estructural, el cual siempre tiene lugar en el universo de las estructuras, ámbito imperceptible a los sentidos humanos, y que solo es comprensible desde la interpretación. Por ello su velocidad, es decir: su forma de desplazamiento en el tiempo, posee escalas diferentes a la existencia humana.

5 Aurell, 2005; Mato, 1994; Ricoeur, 2004.

6 Follari, 1998. 
sucedió al revés, como lo supone la historiografía más tradicional y con ella el discurso oficial que despliegan los Estados con relación a su propia historia, al proponer que la nación, por fuerza, es la entidad que crea a los Estados. Sucede al contrario, como lo ha explicado Eric Hobsbawm, por ejemplo, al afirmar que es el Estado el que crea a la nación. ${ }^{7}$

El pasado colonial en las naciones latinoamericanas muy pronto se transformó en causa justificada de rebelión, y las independencias se volvieron génesis dicientes y manifiestas de la anterioridad de las identidades nacionales. Con el surgimiento de las necesarias Historias Patrias, los procesos independentistas y todos sus avatares fueron interpretados como mitos genésicos, con todo lo que ello sugiere y representa. ${ }^{8}$ Como mito, las independencias se convirtieron en relato de la nación, y más de dos siglos después de aquellos acontecimientos, los discursos historiográficos y oficiales sobre el asunto continúan sosteniendo el mismo sentido justificador y dignificante de la gesta. ${ }^{9}$ Estos discursos son, al mismo tiempo, vehículo y dispositivo reproductor de la eficacia simbólica de esa justificación. La lógica de la nación, aquí como en todas partes, se cumple con todas sus condiciones y complejidades.

Más allá del propio mito, se observa en esta problemática sostenida a través de doscientos años a la estrecha relación que vincula a nación y Estado, nexo indivisible en el discurso moderno sobre la existencia misma y el sentido de sociedad que abrigan ambas nociones. La figura clave del orden social moderno, el Estado nación, supone que al aparato institucional que conjuga a las relaciones de poder que toman decisiones, se le adosa, indefectiblemente, la sociedad sobre la que se levanta como ente regidor y censor. El aspecto fundamental de esta ecuación no es el Estado, obviamente (pueden hallarse rastros milenarios de su existencia), sino la nación, pues en ella recae la novedosa armazón conceptual que la modernidad depositó en su seno como representación de la sociedad. ${ }^{10}$

La historia de la nación es, por tanto, la historia de la identidad, del nosotros que está por encima de la existencia individual, del nosotros ideal que significa la nación misma. Escribir, enseñar y reproducir esa

7 Hobsbawm, 1991. De esa obra, también, procede la siguiente afirmación que se articula coherentemente con lo que aquí planteamos: «...nación y nacionalismo ya no son términos adecuados para describir, y mucho menos para analizar, las entidades políticas que se califiquen de tales, o siquiera los sentimientos que en otros tiempos se describían con ellos». Ibidem, 202.

8 Hemos adelantado una noción de «mito genésico» en Altez, 2011.

9 Quintero, 2011.

10 Anderson, 1993; Breuilly, 1990; Gellner, 1988. 
historia es una misión (formal y oficial) del Estado, el soporte institucional de la nación, y en su reproducción, en tanto que relato, se funda la tradición nacional, el sino de la identidad que articula las subjetividades y las proyecta como colectivo. ${ }^{11}$ En esa misión articuladora, la memoria colectiva juega un papel fundamental: el de unificar al pasado en un sentido único que no puede ser discutido o cuestionado, pues es el sentido del «nosotrossociedad», propiamente.

La memoria colectiva no se encuentra flotando sobre la patria ni se transporta sobre el pensamiento auto-reflexivo de los individuos; por el contrario, los individuos, la sociedad misma, son insertados en esa memoria a través de los recursos de socialización que se imponen desde el Estado. Ningún sujeto, por sí mismo o por el simple hecho de haber nacido en cierto territorio, nace con una memoria en particular; no se trata de un fenómeno asociado a la tierra en la que se viene a la vida, sino de la adquisición de una información que se vuelve referente común con el resto de los individuos con los que convive, la cual es impuesta a través de las estrategias formales de socialización. En ese sentido, la educación formal, vehículo concreto de la eficacia simbólica del Estado, es el instrumento a través del cual se incorporan los individuos a esa memoria colectiva.

Esa «lista de acontecimientos cuyo recuerdo conserva la historia nacional», como ha dicho Halbwachs, «no es ella, no son sus marcos los que representan lo esencial de lo que llamamos memoria colectiva», ${ }^{12}$ no obstante, lo que resulta de ello es lo que finalmente hallará el significado común que se le otorga al imaginario y a la interpretación, también comunes, del pasado nacional, del pasado colectivo. Los hechos que la historia oficial enumera como si se tratase de una concatenación lógica de sucesos que tienen como final el surgimiento de la nación, se transforman en «nuestros hechos», en antecedente directo de nuestra existencia. Eso que Halbwachs llama «historia nacional», no es sino la tradición impuesta desde el Estado, la interpretación única del pasado que viene a servir de antecedente justificativo de la ideología nacional. Su fuerza integradora, centrípeta y excluyente, es el fondo subjetivo de las identidades nacionales, forjado sobre un relato que se vuelve memoria por el efecto de la socialización formal.

Oficial y tradicional, esta historia que decretó a la nación como razón y expresión de las independencias, acabó siendo un discurso hegemónico

11 Vilar, 2004.

12 Halbwachs, 1995, 212. 
e indiscutible,,$^{13}$ solidificado por su propia fuerza centrípeta y arropadora, a la luz de la transparente misión de haber sido (y continuar siendo) el relato de la nación. Ideológicamente legítimo, historiográficamente soportado y metodológicamente confuso, este relato se fundó sobre «una interpretación maniquea de la independencia», donde una nación en ciernes habría despertado de su letargo en opresión para levantarse contra el imperio que la explotaba, desplegando una lucha aguerrida «entre buenos y malos, entre patriotas y traidores, también entre vencedores y vencidos. Construcción de la nación que alumbró la historia patria». Manuel Chust y José Antonio Serrano señalan lo siguiente al respecto:

Un discurso que se volvió hegemónico y que tenía el sentido de unificar la historia de las sociedades altamente diferenciadas étnica y socioeconómicamente, así como con amplios contrastes regionales. [...] Las guerras de independencia interpretadas desde el nacionalismo se convirtieron en sustrato histórico común de las naciones iberoamericanas. Éstas fueron el inicio de su historia contemporánea. Y, en esto, no hay mucha diferencia con la Europa occidental. ${ }^{14}$

El sentido esencial de las memorias colectivas (especialmente en Latinoamérica), reproduce esa esencia justificadora de la nación, convirtiéndole en horizonte simbólico. Para este relato, por ejemplo, la naturaleza es un espejo romántico del ideal telúrico de las identidades, y nada más. ${ }^{15}$ No existe en esta fuerza convocadora de las subjetividades nacionales una relación que advierta a la naturaleza en sus funciones regulares y en su convivencia fenoménica con las sociedades. No hay registros capaces de enlazar a la identidad y la memoria colectiva con su entorno natural más allá de los significados románticos y alucinatorios de costumbre. Ese vínculo está aún por construirse.

\section{Catástrofes en el olvido}

Los desastres son el resultado de procesos materiales y subjetivos que se producen a través del tiempo, o lo que es lo mismo: son el resultado de procesos históricos. ${ }^{16}$ En tanto que puedan ser entendidos de esta manera,

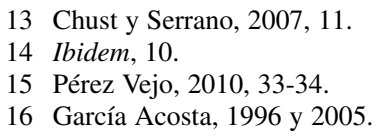


sus manifestaciones dramáticas y paroxísticas comenzarán a ser comprendidas como indicadores de esos procesos, y solo así podrán convertirse en objetos de estudio de las disciplinas históricas, logrando salir del silencio historiográfico al que han sido sometidos. Mientras tanto, desastres y fenómenos naturales permanecerán encarnando los mismos significados de costumbre: sorpresa, interrogantes existenciales, mitos, leyendas, la proyección hacia la naturaleza de las responsabilidades humanas, e inclusive la humanización de sus comportamientos. Con este sentido, no parece posible la incorporación de los desastres a la memoria colectiva como productos concretos de procesos históricos, ni la relación concreta de esas sociedades con la naturaleza que les envuelve en sus lugares de asentamiento. Continuando esto así, los desastres y los fenómenos permanecen sumidos en el olvido característico de aquellas sociedades que reproducen la riesgosa escisión entre naturaleza y cultura.

El olvido es una forma, parafraseando al antropólogo francés Marc Augé, cuyo contenido está determinado por cada proceso histórico y social desde el cual se le da sentido y contenido a esa forma. Olvido y memoria representan una dualidad cuyo significado se apoya en ambas partes, de manera que se envía al olvido aquello que se extrae de la memoria. Ambos, memoria y olvido, constituyen un proceso de selección, pues no es posible recordarlo todo, del mismo modo que no es posible olvidarlo todo. ${ }^{17}$

En esa lógica subyace la relación que una sociedad construye y reproduce con su entorno natural. Lo que generalmente se le sustrae al comportamiento de la naturaleza, es la regularidad de sus fenómenos, nublando la relación de convivencia que indefectiblemente existe entre las propias sociedades y esos fenómenos naturales. En este sentido, no solo es el temor al dolor y la destrucción vivida con cada catástrofe lo que impulsa al mecanismo de defensa, sino la construcción sistemática de una memoria colectiva que a partir de la contemporaneidad moderna se instruye desde los intereses del poder.

Solo puede olvidarse aquello que ha sucedido. No obstante, el olvido no necesariamente es un acto voluntario, decretado, sino un resultado. Freud lo explicó con mayor precisión y propiedad: «en la vida psíquica nada de lo una vez formado puede desaparecer jamás...». ${ }^{18}$ Esto permite razonar que lo que se olvida solo se deposita en algún lugar, guardándole 
de la posibilidad de entrar en contacto con la realidad sensoperceptible. He allí que olvidar implique construir una realidad paralela a la percibida, una realidad que pretende interponerse ante la que se construye diariamente. Este mecanismo que descompone la percepción de la propia realidad eventualmente opera para olvidar los desastres.

En ocasiones, eludimos el sufrimiento que puede acusar la memoria tratando de no recordar la que puede herirnos. El olvido, en este caso, resulta activo. Tiene lugar sobre todo en el plano de la historia y de las grandes catástrofes históricas... ${ }^{19}$

La vulnerabilidad ante los fenómenos naturales potencialmente destructores comienza por el desconocimiento de su presencia regular, y esto, a su vez, inicia en el olvido que se ha construido desde una memoria colectiva que solo da la cara a la historia política y heroica, legitimadora y cómplice de poderes y gobiernos.

Cuando esos fenómenos son eventualmente incorporados, por lo general se asumen desde interpretaciones alucinatorias o veladoras de sus reales efectos. Esas incorporaciones a la memoria colectiva con la que se distinguen algunos desastres, o bien algunos fenómenos recurrentes, padecen de distorsiones o bien son recreadas desde el imaginario colectivo, el cual también se halla intervenido por procesos de resignificación del pasado, a menudo impulsados desde la propia nacionalización de las memorias, así como desde procesos contemporáneos inductores de ese imaginario.

Si la memoria fuese un acto mecánico, poco habría que agregar acerca de las representaciones que los fenómenos naturales construyen dentro de las estructuras simbólicas de la sociedad. Sin duda, sus irrupciones y efectos se convertirían en referentes indefectibles, pues el asentamiento concreto de una sociedad sobre su entorno natural se encuentra determinado por las regularidades fenoménicas y los ciclos de la naturaleza, aspectos que de por sí habrían de conducir a la construcción de una relación indivisible entre medioambiente y sociedad. Pero la memoria es un depósito de sensaciones que acumula respuestas ante hechos que se atesoran sin que muchas veces se esté plenamente consciente de ello. Esas respuestas, eventualmente transformadas en reflejos casi musculares, cuando se trata de la memoria colectiva de una sociedad, pueden estar condicionadas por la forma a través de la cual se han ido almacenando esos hechos. Y esto no depende siempre de la voluntad de la sociedad (tal como si ese colectivo

19 Paul Ricoeur, citado por Nieto López, 2006, 88. 
operase al igual que un individuo o poseyese una personalidad similar a la de un sujeto), sino de las estrategias con las cuales se socializó a ese colectivo. En ello han participado todos los componentes de una sociedad, desde el Estado formador y educador, hasta la actividad de las propias comunidades con sus recursos de supervivencia. ${ }^{20}$

Por consiguiente, las memorias colectivas que se han construido desde la función socializadora de los Estados nacionales, al menos en el caso latinoamericano, no solo han desdibujado a la naturaleza y sus regularidades detrás de la nebulosa y «telúrica» idea de la nación, ${ }^{21}$ sino que al mismo tiempo han nacionalizado esas memorias, de manera que los pasados solo alcanzan a ser comunes a los colectivos que conviven dentro de las fronteras impuestas por los movimientos nacionales. La nacionalización de las memorias, en consecuencia, ha levantado fronteras simbólicas e históricas sobre las interpretaciones de los pasados regionales, ambientales y fenoménicos, diluyéndoles tras los discursos justificadores de la nación. Esta problemática resulta más crítica aún en las regiones propiamente fronterizas, donde las memorias tropiezan con las arbitrariedades limítrofes y limitantes de la «patria».

Allí, los fenómenos naturales, destructores o benéficos para las comunidades de la zona, van y vienen con la cotidianidad, viéndose relegados a formar parte de un entorno que se antoja esencialmente diferente a la condición humana, apeados definitivamente de la memoria colectiva por los procesos formales de socialización, y arrojados al olvido sistemático del relato identitario nacional: la naturaleza es un vecino incómodo, especialmente cuando se manifiesta en forma de fenómeno destructor, y en poco o

20 Con todo, parece pertinente agregar que las cuestiones vinculadas al arraigo, por ejemplo, o bien los afectos que representan un vínculo subjetivo con el lugar de socialización de los individuos, continúa siendo un problema interpretativo sugerente e intrincado para las disciplinas que, como la antropología, todavía no hallan para esos casos una respuesta consensuada o teóricamente satisfactoria, a pesar de las generalidades que caracterizan a sus discursos al respecto.

21 Quizás exista una respuesta a las preguntas antropológicas sobre el arraigo y ese vínculo afectivo anclado en la idea de «terruño», o bien de «país», como se ha llamado de antiguo al lugar de nacimiento u origen de los individuos. La noción de «telúrico» que utilizamos aquí proviene de esa idea romántica y seductora que los discursos forjadores y reproductores de la nacionalidad han utilizado desde siempre, y que parece convencernos de que, en efecto, existe un afecto natural que proviene de la «tierra», esa palabra con asociación etimológica a la voz latina «patria», a través de lo cual habría de fundarse un nexo inconmovible entre los sujetos y el entorno en el que nacen. Una arqueología de estos conceptos ha de hallar, de seguro, una sinonimia históricamente construida entre las voces país, patria, tierra y nación, algo que ha entrampado a la antropología en su idea de identidad. Si todo esto operara de manera natural, entonces, y en buena medida, lo que planteamos aquí en este trabajo no tendría lugar. 
nada contribuye a la memoria colectiva hecha de lógicas nacionales. La recurrencia de los desastres en las regiones que padecen este tipo de fenómenos con regularidad, no necesariamente conduce al aprendizaje de la coexistencia sociedad-contexto natural, ni a la experiencia suficiente como para desplegar gestiones de riesgo exitosas. ${ }^{22}$

Por lo tanto, con cada terremoto, erupción, alud torrencial, sequía o fenómenos de gran extensión como El Niño, los recursos utilizados se manifiesten en forma de asistencia, atención a la emergencia y reconstrucción, pero nunca se levantan en forma de prevención real, pues ello habría de indicar el reconocimiento de sus regularidades, o bien el testimonio fehaciente del conocimiento del pasado. Y ese conocimiento, funcionalmente reservado para la historia de la nación es, antes bien, una carencia tan común como su aspecto contrario: la ideología nacional.

\section{Desastres con pasaporte $y$ sin visa}

Las naciones latinoamericanas demarcaron sus límites sobre las antiguas jurisdicciones coloniales. Por lo general, sus fronteras proceden de acuerdos o conflictos binacionales que resultan de las disputas sobre las posesiones territoriales de provincias y virreinatos del pasado. Sin embargo, las fronteras provinciales del período colonial no significan ni representan lo mismo que las nacionales. Aquellas jamás se plantearon encerrar identidades ni defender soberanías, sino delimitar los dominios ultramarinos peninsulares desde su función propiamente administrativa. No construyeron, a despecho del romanticismo moderno, ningún «apego al terruño»o sentido de patria, como lo han hecho creer desde el relato nacional. Las sociedades que se conformaron al calor del proceso colonial se articularon históricamente en torno a la dinámica regional-geográfica y jurisdiccional del sistema metropolitano español. Hoy sus referentes se hallan perdidos y confundidos con la mitología nacional. Allí, en ese pasado temporalmente cercano y simbólicamente distante, la relación de las comunidades con la naturaleza y sus fenómenos encerró significaciones que en el presente se desconocen, y que la nacionalización de las memorias se ha encargado de sepultar debajo de su relato de auto-justificaciones.

22 Revet, 2007, 35.

Anu. estud. am., 73, 1, enero-junio, 2016, 319-350. ISSN: 0210-5810. DOI: 10.3989/aeamer.2016.1.11 
Los desastres del pasado colonial, al igual que su proceso histórico, solo pueden ser históricamente reconstruidos desde los esfuerzos metodológicos del presente. Todo lo que se puede saber de ese pasado se filtra en el tamiz de una historiografía más nacionalista que nacional, ${ }^{23}$ preocupada por reproducir la secuencia de los héroes en la construcción de la nación, y concentrada en las variables historiográficas de siempre: lo político, lo económico y lo social. La historia de la naturaleza, sus fenómenos y sus efectos en la vida social, económica y política de estas sociedades, no ha sido un objeto de estudio ni un objetivo de comprensión en esa historiografía que tropieza su mirada y sus fuentes contra las fronteras nacionales.

Con ello, las fuentes primarias, documentales y testimoniales sobre el pasado (colonial o republicano), yacen al abrigo de repositorios cuyas jurisdicciones son ahora nacionales, independientemente de que su origen provenga del orden colonial-provincial. La información sobre los hechos de ese pasado pre-nacional, se disemina entre archivos y fuentes sometidos a jurisdicciones soberanas que acaban por fragmentar a su vez a la memoria. Esto afecta, desde luego, a la reconstrucción de esos pasados y a su comprensión, y con ello al conocimiento certero de los hechos, como los desastres o los efectos regulares de los fenómenos naturales conducentes a padecimientos extremos.

Todo lo que en el pasado colonial haya tenido lugar sobre las actuales fronteras nacionales, queda dividido entre dos memorias, cuando menos, que se dan la espalda, y el único camino para reconstruir sus procesos es el de la búsqueda complementaria a ambos lados de las fronteras. De allí que los desastres del pasado, por lo general, registran efectos que se atenúan o desaparecen conforme se aproximan a los límites de la actualidad. Aquellos que alcanzaron a afectar las regiones a ambos lados de los márgenes presentes, de seguro cuentan con impactos y efectos lo suficientemente graves y contundentes como para haber generado registros documentales sobre grandes extensiones geográficas. Sin embargo, en el caso de fenómenos con efectos de menor rango de alcance, la información producida suele dividirse por localidades, descubriéndose al presente en forma atomizada dentro de los archivos parroquiales o regionales..$^{24}$ De esta manera, los desastres que se hallan fragmentados entre legajos esparcidos de forma aislada por archivos de países diferentes y que hoy aparecen como

23 Cardozo, 2005, 9.

24 García Acosta, 2001 y 2004. 
eventos distintos o inconexos, de seguro han de representar una mayoría declarada.

De ello dan cuenta los esfuerzos que al presente realizan algunas instituciones dedicadas a la investigación de ciertos fenómenos y sus efectos sobre la sociedad, las construcciones y la naturaleza, como los observatorios sismológicos o vulcanológicos. Y los ejemplos de eventos históricos cuyos efectos parecen desaparecer al topar con las fronteras nacionales son elocuentes al respecto. Esto se vuelve más problemático cuanto más hacia el pasado se trasladan las fechas.

Como casos característicos del asunto pueden citarse algunos eventos que han tenido lugar sobre las fronteras entre las actuales Colombia y Venezuela. Por ejemplo, el sismo del 3 de febrero de 1610, que causó daños en las localidades hoy fronterizas de La Grita y San Cristóbal en Venezuela con graves efectos sobre la geomorfología de la región, ${ }^{25}$ parece desaparecer en sus registros hacia los pueblos de la actual Colombia, unos pocos kilómetros al Oeste. Otros casos del pasado profundo colonial presentan confusiones, como sucede con el terremoto que el 16 de enero de 1644 sacudió a Pamplona (Colombia), al que se la ha atribuido erróneamente la destrucción de Mérida (Venezuela), por confundirle con el terremoto que, con la misma fecha pero en 1674, sí causó daños en la ciudad hoy venezolana. ${ }^{26}$ Casos como estos abundan en el período colonial de esta zona fronteriza, y las historiografías nacionales reproducen los mismos errores al respecto sin la menor preocupación por el asunto.

Más recientemente, en el período republicano, temblores que han estremecido localidades en la misma frontera enseñan daños atenuados o fragmentados por la misma línea divisoria entre ambos países. Este es el caso del terremoto del 18 de mayo de 1875 , bautizado como «Sismo de Cúcuta» (por haber sido la ciudad más afectada), cuyos efectos parecen atomizarse sobre localidades que se derraman a ambos lados de la frontera, sin que hasta el momento se haya realizado una investigación conjunta entre ambos países, o bien un estudio que contemple archivos y repositorios en las dos márgenes de la frontera. El Sismo de Cúcuta es un ejemplo característico de la «nacionalización» de los fenómenos.

Erupciones y temblores reproducen la misma problemática en otras zonas fronterizas latinoamericanas. Por ejemplo, ¿cuánto podría conocerse

25 Ferrer y Laffaille, 1998.

26 Altez, 2010; Palme y Altez, 2002. 
del terremoto del 23 de mayo de 1575 que afectó a San Salvador, pero que tuvo efectos en Guatemala y Chiapas, si se revisaran las fuentes primarias que cubren esta zona, hoy entre tres países? Algo similar podría decirse de los temblores que han afectado a Arequipa, en el actual Perú, hacia 1554, $1582,1599,1600,1604,1716,1725,1738,1784$ y 1821, por citar aquellos con pasado de fronteras virreinales y presente de fronteras nacionales que afectan a tres países: Perú, Bolivia y Chile. ${ }^{27}$

El caso de los sismos y los volcanes permite una búsqueda puntual de sus efectos por hallarse impulsados a partir de una o varias fechas específicas (las del temblor o las de las erupciones), y en ese sentido la prosecución histórica es eventualmente más accesible, a pesar de las dificultades señaladas. No sucede lo mismo con los fenómenos de impacto lento o de efectos sostenidos, como las sequías o las anomalías meteorológicas. Ausencia de precipitaciones que se extienden por varios años dificultan su precisión en el tiempo, y si su alcance abarca largas regiones que hoy se encuentran divididas entre países, la interpretación del fenómeno como un solo evento de gran extensión territorial resulta ser un problema para la investigación del caso, y casi una utopía si se piensa en reconstruirlo como memoria colectiva en su zona de impacto.

Fenómenos como el ENSO ${ }^{28}$ de extensiones continentales y de largo alcance temporal en sus efectos, han demostrado que las sociedades expuestas a su retorno ofrecen una memoria colectiva focalizada, en el mejor de los casos, o bien memorias fragmentadas y disociadas regionalmente que dan cuenta con mayor énfasis de las vulnerabilidades de esas comunidades, que de sus fortalezas al respecto. A pesar de la existencia de comunidades que han sostenido a través del tiempo ciertas características o prácticas que indican adaptabilidad a su medio y al clima en el que se insertan (como algunas comunidades centroamericanas y mexicanas), ${ }^{29}$ tanto esas prácticas como su memoria al respecto se encuentran hoy intervenidas por procesos nacionales (o transnacionales) que modifican (o son capaces de modificar) sus tradiciones.

Sin embargo, no todas las comunidades que se agrupan hoy bajo sociedades nacionales provienen de pasados precolombinos, y no son ellas tampoco las únicas con tradiciones vinculadas al medioambiente, independientemente de que esas tradiciones supongan inadaptaciones o la

27 Para saber más de estos eventos, se sugiere consultar la obra de Petit-Breuilh, 2004a.

28 «l Niño Southern Oscillation», por sus siglas en inglés.

29 Ver los trabajos de Briones, 2012; y Audefroy, 2012. 
potenciación de las amenazas con las que conviven. Pensamos que estas comunidades, forjadas en el pasado colonial, en su mayoría, o en procesos republicanos y modernos, han padecido también la intervención de los procesos formales y oficiales de socialización, y con ello, desde luego, han asistido al modelamiento de sus memorias colectivas. Creemos que no existe, al presente, ninguna comunidad con asiento en Latinoamérica que no se encuentre articulada simbólica o ideológicamente con los procesos de nacionalización de las memorias y las identidades.

Los ejemplos son numerosos desde México hasta el extremo sur de América, y enseñan con ello la calidad de la problemática, así como la escasa atención hacia la historia de los fenómenos naturales y su relación indivisible con la sociedad. Demuestran, a su vez, que la importancia de las memorias colectivas en estos países descansa sobre la nacionalización de sus pasados, resquebrajando los procesos históricos en impertinentes divisiones territoriales que desarticulan, fragmentan e ideologizan los procesos simbólicos de las memorias colectivas regionales y, especialmente, las relaciones de integración e incorporación de la naturaleza a los procesos sociales.

\section{Memorias que no encajan}

Las naciones que se independizaron de la corona española en el siglo XIX dibujan continentes divididos en países que poco tienen que ver con el sentido de las fronteras provinciales del período colonial. Si algunas líneas coinciden físicamente, no significa que representen lo mismo simbólicamente. Sin embargo, sobre estas fronteras impuestas por las divisiones territoriales nacionales, se han levantado memorias que fragmentan los procesos subjetivos e históricos que se fraguaron desde el siglo XVI en esas regiones.

Estos procesos de nacionalización de las memorias colectivas, como se ha apuntado anteriormente, interrumpen los procesos simbólicos de la sociedad colonial, resignifican y resemantizan los procesos de integración regional, y fragmentan los procesos de conformación de memorias colectivas originados siglos atrás. Al presente esas memorias, intervenidas por la socialización formal de los Estados nacionales, no encajan entre sí, y eventualmente se oponen, antes que complementarse simbólicamente.

Fronteras nacionales en disputas conforman, además, procesos de desarticulación social en torno a las líneas imaginarias que imponen 
soberanías sobre las subjetividades regionales. Incluso las relaciones de parentesco, de asociación, de cotidianidades, de intercambios comerciales, de circuitos pastoriles, o de simple circulación, se ven interrumpidas y flanqueadas por intereses de poder que diluyen los procesos centenarios de integración regional.

Mayor problemática, además, se conjuga en regiones fronterizas sobre las que se asientan comunidades indígenas o conflictos armados por el narcotráfico, la guerrilla o la delincuencia común. Estas zonas, complejas por las condiciones que presentan en la actualidad, suponen dificultades aún más graves al tratar de reconstruir su pasado (reciente o lejano) en torno a su relación con la naturaleza y sus manifestaciones fenoménicas. Aquí la nacionalización de las memorias es una traba que se superpone a las conflictividades existentes, y que en casi todos los casos acaba por agravar las circunstancias.

La mayoría de los países latinoamericanos cuentan hoy con fronteras en disputas, e incluso muchos de ellos han llegado a la confrontación bélica. Buena parte de esos países, enemistados políticamente por sus fronteras o por enrevesadas diferencias ideológicas, comparten zonas expuestas a amenazas naturales conducentes a riesgos de envergadura, como los sismos, los volcanes, las anomalías climáticas, entre otras variables que afectan directamente sus medioambientes. Las fronteras de la región Andina son el más claro ejemplo al respecto: Chile, Argentina, Bolivia, Perú, Ecuador, Colombia y Venezuela resumen estos problemas en la historia de su existencia como naciones independientes.

Los fenómenos naturales cuyos impactos y efectos favorecen los desenlaces catastróficos, y que son compartidos entre esas regiones fronterizas, son capaces de desnudar las vulnerabilidades de esas sociedades de manera transversal. Sismos, erupciones, sequías, lluvias, anomalías meteorológicas, e inundaciones, por ejemplo, estremecen comunidades y formas de vida en casi todos esos territorios. Selvas, bosques, ganado, recursos materiales, vida comunitaria y cotidianidad, acusan consecuencias que son independientes de las líneas divisorias impuestas por las realidades nacionales, pero que precisamente por ellas ven incrementadas las afecciones de esos fenómenos tal como si las circunstancias de cada país se convirtieran en amplificadores o atenuadores de estos efectos, según sea el caso.

Cuando esas amenazas se prolongan en el tiempo, como sucede con los fenómenos cuyo retorno supera grandes lapsos (décadas e incluso siglos), la ausencia de memorias colectivas que sostengan la advertencia de 
esos retornos, puede contribuir con desenlaces desastrosos o superar la capacidad de respuestas a las emergencias que han sido preparadas en tiempos modernos y cercanos. Esto sucede, por ejemplo, con el caso del ENSO en las comunidades de los Andes, y su facilidad para concatenarse con otras amenazas a través de las largas exposiciones a sus efectos, por tratarse, precisamente, de fenómenos capaces de mantener su impacto por periodos extensos. ${ }^{30}$ Esas respuestas modernas, desde luego, han sido promovidas y puestas en práctica, con o sin éxito, desde proyectos nacionales o gubernativos, generalmente asociados a intereses clientelares o de inversiones privadas. Tal cosa solo puede atender el problema dentro de sus fronteras, espacio que marca el alcance jurisdiccional de esas intervenciones.

Incluso los procesos de adaptación al clima y al medioambiente, forjados a través del tiempo e inductores de cambios adaptativos en las tradiciones (o bien que han introducido nuevas tradiciones al respecto), como lo notaron los estudios de Amos Rapoport o Paul Oliver, y que se reflejan en viviendas que demuestran la plasticidad cultural de algunas comunidades en su relación con la naturaleza, han sido o son susceptibles de ser intervenidos (en la mayoría de los casos latinoamericanos), por procesos centralizados de planificación urbana o por esas relaciones clientelares que mencionamos. ${ }^{31}$ Estas intervenciones, promovidas desde intereses políticos o inversionistas, eventualmente imponen nuevos sentidos de lo tradicional, a partir de discursos ideológicos que determinan qué es tradición y qué no lo es, dependiendo de los intereses de turno. Sabemos de estos casos en la actual Venezuela, por ejemplo, y con relación a comunidades indígenas asentadas en zonas fronterizas, o bien comunidades denominadas ambiguamente como «campesinas», en la región andina de ese país.

Probablemente, una aproximación a las expresiones más características de las memorias colectivas de las comunidades que se asientan sobre las fronteras latinoamericanas y que conviven con todo tipo de riesgos, dé cuenta de contradicciones, complementos, olvidos e interpretaciones que han sido intervenidas por los procesos de nacionalización de las memorias y por la intervención clientelar de sus tradiciones, igualmente. Con ello, desde luego, el pasado se vuelve una línea que tiene un antes y un después de la independencia, partiendo en dos el significado de los procesos y enviando a un foso inalcanzable e inaccesible los significados construidos

30 Véase Moseley, 2001, 193.

31 Rapoport, 1969; Oliver, 1978. 
en el pasado colonial. Qué decir de aquellos significados que provienen de tiempos precolombinos. ${ }^{32}$

El resultado de esta fragmentación de los procesos simbólicos iniciados en ese pasado colonial o precolombino, y la nacionalización de las memorias colectivas, sin duda ha de conducir a la parcelación de las interpretaciones sociales y culturales sobre los procesos naturales y fenoménicos en esas comunidades fronterizas. $\mathrm{O}$ bien a la fractura de interpretaciones que pueden ser compartidas por tales comunidades, para sumirlas en resemantizaciones con arreglo a fines ideológicos, nacionales o clientelares. Por todos lados, los procesos simbólicos en sociedades asentadas hoy en regiones fronterizas se ven abruptamente intervenidos por las fuerzas centrípetas y excluyentes de los procesos ideológicos nacionales.

\section{Amenazas fragmentadas y reproducción del riesgo}

En tanto que nacionales, las historias de los países latinoamericanos han fragmentado la comprensión de los procesos históricos regionales y con ello la incorporación de interpretaciones también regionales acerca de esos procesos. Se trata de una atomización simbólica que interviene de manera abrupta la incorporación social-regional-comunitaria de las experiencias del pasado lejano o cercano a las memorias colectivas, que acaba escogiendo qué incorporar o qué obviar dentro de esas interpretaciones, así como imponiendo dispositivos interpretativos ya codificados desde los centros de poder y socialización nacionales. Esta atomización del proceso no solo afecta a las memorias colectivas con relación al devenir comunitario-social-

32 A pesar de que los trabajos antes citados de Briones, 2012, y Audefroy, 2012, dan cuenta de adaptabilidades sostenidas en el tiempo por comunidades de ancestralidad precolombina, estos casos representan no solo excepciones, sino también situaciones que no se hallan exentas de la intervención. Como lo indica Audefroy, «la introducción de la llamada modernidad en la vivienda tradicional ha cambiado materiales, sistemas constructivos, formas y hasta modos de vida» $(2013,104)$; o como se pregunta Briones ante la aplicación de medidas de adaptación inducidas desde planificaciones gubernamentales: «Uno de los riesgos es convertir la adaptación en un paradigma comparable con lo que fue el desarrollo de la posguerra. ¿Y si en 30 o 40 años descubrimos que las medidas de adaptación no funcionan? Quizá uno de los principios para el diseño de adaptaciones exitosas consiste en reconocer que las sociedades, pese a muchas limitantes estructurales, no son pasivas frente a sus entornos, lo cual no quiere decir que todas sus prácticas y saberes tradicionales sean siempre convenientes, pero pone en el centro del debate la adaptación proactiva de las comunidades frente a la lentitud de las políticas públicas limitadas hasta el momento en protocolos internacionales que tomarán varios años en tener impactos en la escala local» $(2012,112)$. 
regional, sino también a las formas de comprender y de adaptación de esas sociedades con relación a sus medioambientes y entornos naturales.

Regiones, comunidades y sociedades que conviven con amenazas naturales asociadas a fenómenos de regular comportamiento, han acoplado sus formas de interpretación y adaptación a sus entornos con los mecanismos de socialización nacionales que se imponen formalmente desde los centros de control y de poder de cada país. Esto ha afectado tanto a las comunidades asentadas en zonas alejadas de las fronteras territoriales, como a aquellas que se encuentran sobre los límites nacionales. En estos casos, la intervención sobre los procesos simbólicos vinculados a esa convivencia con los fenómenos naturales potencialmente destructores, como ya se ha mencionado, fractura las posibles articulaciones comunitarias o regionales que se superponen a las jurisdicciones nacionales.

Al fragmentar los procesos subjetivos y simbólicos de convivencia e interpretación de esos fenómenos y de los entornos medioambientales, las comunidades asentadas sobre regiones fronterizas nacionales acaban por articularse con los procesos simbólicos e ideológicos construidos desde los centros administrativos de poder, interviniendo con ello a los procesos de memorias colectivas que venían de antiguo. La proximidad a las amenazas naturales, en estos casos, se traducen en probabilidades heterogéneas de riesgos que dependen de las eficiencias o ineficiencias de los Estados que atienden estas regiones y estas comunidades.

Por lo general, las regiones fronterizas representan un conglomerado de problemas para los Estados nacionales (y en especial en los casos latinoamericanos), que históricamente han obviado a las amenazas naturales de sus agendas de turno. Solo después de que la Organización de Naciones Unidas hizo del tema una prioridad a partir de la última década del siglo XX, los riesgos y sus probables consecuencias se colocaron en esas agendas, con lo que, eventualmente, el problema fue atendido de manera circunstancial. Un proceso de especialización institucional y crecimiento discursivo sobre el asunto permitió que los Estados latinoamericanos (fundamentalmente) atendieran el tema, ya de la mano de experiencias desastrosas, o bien desde el impulso generado por el conocimiento profesional. ${ }^{33}$

Con todo, la atención al caso (salvo aisladas experiencias regionales) acabó siendo nacional, de manera que las regiones fronterizas y sus convivencias con las amenazas (como sucede en la región Andina, como

33 Petit-Breuilh, 2004a, 13-15. 
ejemplo característico), han dependido de esas eficiencias o ineficiencias nacionales para sus estrategias de prevención de riesgos. Un caso excepcional lo representa el Proyecto PREDECAN (Prevención de Desastres en la Comunidad Andina), que de la mano de la Comunidad Andina de Naciones (CAN) y con patrocinio de la Comisión Europea, elaboró entre los años 2005 y 2009 un programa conjunto de atención a los riesgos en las naciones de la comunidad que arrojó productos comunes. ${ }^{34}$ Como una muestra de las problemáticas ideológicas y políticas mencionadas, hay que subrayar que Venezuela, en una acción de manifiesta distancia ideológica con la CAN, se retiró del proyecto en el año 2007 y quedó por fuera de sus resultados. ${ }^{35}$

De esta manera, regiones y comunidades fronterizas ubicadas en zonas de alcance o manifestación de ciertos fenómenos conducentes a padecimientos extremos, pero distribuidas sobre países diferentes, acaban por convivir con una misma amenaza que fragmenta sus riesgos de acuerdo a las condiciones y formas de respuestas que ofrece cada país en particular. Un ejemplo de esto tiene lugar con los volcanes ubicados entre Argentina y Chile, y basta con referir las erupciones del Lonquimay entre 1988 y 1989. Por entonces los chilenos padecían el régimen de Pinochet, y en la intervención de la emergencia se ha conocido, tiempo después, que las enfermedades asociadas a las emisiones de gases o a la exposición al flúor no se hicieron públicas, como tampoco se revelaron las potencialidades de contaminación estimadas por las autoridades, información que tampoco fue conocida en Argentina, con zonas habitadas próximas al lugar. ${ }^{36}$

Los riesgos se producen y reproducen histórica y socialmente, ${ }^{37}$ y las probabilidades que se desprenden de estas situaciones y circunstancias se verán condicionadas por las atenciones particulares sobre el asunto que

34 http://www.comunidadandina.org/predecan/. Fecha de consulta: 10 de octubre de 2013.

35 Hemos tenido la oportunidad de participar en el proyecto como representante de Venezuela en «Taller Subregional Andino para la discusión de conceptos y enfoques en Gestión de Riesgo, Prevención y Atención de Desastres / Protección Civil y para la definición de metodologías de referencia común para la formulación de planes de Gestión Local de Riesgo» (La Paz, Bolivia 2006), y en otros talleres de PREDECAN llevados a cabo en Caracas, desarrollando la Mesa de Trabajo n. ${ }^{\circ}$, «Fortalecimiento del Conocimiento y la Investigación». En mi caso particular, fui el único delegado ante el proyecto que no provenía del gobierno nacional, sino de instancias académicas. La participación de Venezuela se retiró a comienzos del año 2007, impidiendo con ello la continuidad de las propuestas desplegadas en esos talleres.

36 Petit-Breuilh, 2013.

37 García Acosta, 2005; aunque la autora utiliza el término «construcción» y no «producción», llega esta misma conclusión: se trata de procesos históricos y sociales. 
cada país despliegue al respecto. Una misma amenaza ubicada sobre una región fronteriza se verá fragmentada por esa misma situación, produciendo y reproduciendo los riesgos de acuerdo a las condiciones que cada país ofrezca con relación al problema. La fragmentación de las amenazas supone, en todo caso, una reproducción heterogénea de los riesgos que, al atender el problema de esta manera, vuelve ineficiente toda preparación parcelada de tal situación.

Una misma amenaza sobre este tipo de regiones, en consecuencia, representa la reproducción heterogénea y transversal de los riesgos en esos territorios y sobre esas comunidades, contribuyendo de esta manera a hacerlas más vulnerables. Se trata, pues, de contextos vulnerables con variabilidad nacional-regional, cuya condición de vulnerabilidad no solo se hace evidente en aspectos materiales, infraestructurales, de preparación, o atención a posibles emergencias, sino también desde lo simbólico y lo subjetivo, pues sus memorias (fragmentadas, intervenidas por la ideología nacional, y de corto alcance temporal), apenas pueden incorporar a su interpretación y adaptación ambiental la convivencia con fenómenos naturales potencialmente destructores de regular comportamiento. ${ }^{38}$

\section{Reconstruir memorias y borrar fronteras para reducir riesgos}

Las fronteras tienen su historia, y a menudo son fundidas en clave de elementos emblemáticos con las historias de las naciones. Se confunde en ello a la jurisdicción con la soberanía y al territorio con la identidad, en una muestra más de la eficacia simbólica de la ideología nacional. Pero las historias de las fronteras y sus vicisitudes políticas, no necesariamente son las historias de las sociedades que habitan en ellas, y mucho menos las historias de esas sociedades en convivencia con su medioambiente y los fenómenos naturales. Allí la naturaleza apenas es un accidente geográfico que sirve de marmolillo demarcador.

Sin embargo, la naturaleza y sus fenómenos siguen allí y continúan su regularidad, junto con sus vecinos humanos, haciendo una historia que en

38 El efecto que la aplicación de políticas y programas preventivos o mitigadores del riesgo ha de causar en estas regiones, está por verse, y quizás en unas cuantas décadas podremos observar con mayor detalle cómo ha de producirse, reproducirse, y seguramente transformarse la relación subjetiva de estas comunidades con el medioambiente, o bien cómo ha de profundizarse la cortedad de sus memorias y su escasa capacidad de adaptación. El proceso está en pleno desarrollo, en todo caso. 
muchos casos está por escribirse, especialmente con relación a las regiones fronterizas, donde el tiempo y la memoria se han visto fragmentados por las fuerzas centrípetas de los poderes nacionales. Planteada esta problemática en los párrafos antecedentes, nos proponemos llamar la atención al respecto y tomar en cuenta algunas herramientas alternativas para confrontar el asunto en búsqueda de recursos eficientes y soluciones posibles, advirtiendo que cuando esa convivencia con la naturaleza halla como protagonistas a ciertos fenómenos conducentes a padecimientos extremos, dichos fenómenos se transforman en amenazas y sus manifestaciones se revisten de riesgos que dejan de ser característicamente regionales para volverse arbitrariamente nacionales, binacionales, o incluso multinacionales.

A pesar de que la memoria no es una «cosa» que pueda transformarse a voluntad, parece posible aportar elementos que permitan reconstruirla. En este punto resulta pertinente precisar que reconstruir no es lo mismo que rescatar. El «rescate» de la memoria (a menudo promovido por la antropología de corte populista y romántico), supone, a grandes rasgos, la captación de información que por lo general es tomada a través de entrevistas orales, y si pensamos que la memoria colectiva está hoy determinantemente intervenida por su nacionalización, esa recuperación de la información (vinculada a la historia de la relación que esas comunidades ha sostenido con la naturaleza en sus regiones de asentamiento), ha de encontrar un imaginario distorsionado en lugar de una memoria eficiente. Por eso creemos que la reconstrucción, que entendemos como el entrecruzamiento complementario de la información histórica, la científica especializada, la ambiental y la oral, es el objetivo que podría contribuir con la producción de nuevos mecanismos adaptativos al respecto.

En el caso de los procesos históricos que se articulan entre sociedad y naturaleza, prácticamente todo está por hacerse. En ese sentido, las memorias colectivas, intervenidas por los procesos ideológicos nacionales, y constituidas por la omisión característica sobre la relación naturalezasociedad, pueden convertirse en herramientas eficaces a partir de la reconstrucción de la información histórica, de la mano de todas las herramientas metodológicas que se complementen al efecto.

El principio fundamental que soporta estas nociones se asienta en la convicción de que es posible convertir al conocimiento histórico en memoria,$^{39}$ a través de estrategias de acceso y divulgación de la información que

39 Altez, 2005, 315. 
involucren a las comunidades y sociedades en general de manera activa, y no solo como entes receptores de la información. Se trata, también y entre otras cosas, de recuperar información histórica desde las fuentes primarias hasta las fuentes tradicionales, y de involucrar a las comunidades en ese proceso de reconstrucción. Al mismo tiempo, se entiende que dicho proceso debe tornarse en una recuperación dinámica, si se incluye en el mismo al contraste de la información hallada con la memoria existente, las tradiciones, el olvido y las relaciones que en la actualidad se despliegan en esas sociedades con sus medioambientes.

Como recurso base puede tomarse a la elaboración de catálogos de eventos adversos vinculados a amenazas naturales que han sido desarrollados desde experiencias nacionales, académicas e institucionales. La catalogación, de por sí, supone la recuperación de toda la información existente y por construir sobre un tipo de fenómeno en especial, y ya con ese producto acabado, el recurso de la contrastación con los saberes y la información que al respecto posean las comunidades en cuestión, conduce a un proceso dinámico de reconstrucción de la memoria colectiva.

La catalogación se figura como una plataforma de información transversal, que incluye desde la documentación primaria hasta la iconografía existente. Del mismo modo, es una fuente esencial para la investigación de esos fenómenos, de manera que el complemento de este producto viene a dar en estudios transversales (históricos, sociales, analíticos, disciplinares) sobre los fenómenos en especial, así como sobre la relación entre sus manifestaciones y efectos con la sociedades que conviven con ellos. Estos resultados contribuyen a enriquecer las formas de interpretación que esas comunidades poseen sobre esta convivencia.

Como aporte metodológico a este trabajo de recuperación de la información histórica, así como a la investigación transversal que se desprende de ello, se sugiere la incorporación de las comunidades al proceso analíti$c o$, desde la propia recuperación de la información (especialmente la oral y la tradicional), hasta el diálogo en la construcción de resultados interpretativos sobre el asunto.

$\mathrm{El}$ aspecto fundamental que se advierte se centra en el hecho de que las regiones fronterizas ofrecen, de suyo, un espectro de problemas concretos que dificultan el desarrollo de este tipo de trabajos. Operar con comunidades asentadas en regiones por el estilo supone, por un lado, un problema político-institucional, pues los trabajos de campo tropiezan con el libre desenvolvimiento a un lado y otro de los límites nacionales. Por otro lado, 
el trabajo con las fuentes primarias no necesariamente conduce a una investigación documental in situ, pues las centralizaciones administrativas de los Estados nacionales han concentrado bajo su injerencia, a su vez, a los repositorios documentales y la información histórica correspondiente a los territorios de cada país. Con ello, los archivos más substanciosos, por lo general, se encuentran a distancia de las localidades ubicadas en las regiones de interés.

Sobre la base del reconocimiento de esas realidades, la catalogación resultaría viable si se acometen proyectos binacionales, multinacionales o regionales, a partir de los cuales se construyan perspectivas integradas sobre las amenazas naturales en regiones fronterizas, con esfuerzos estratégicos y metodológicos cuyos enfoques se asienten en la reducción de los riesgos. Desde luego, para ello se necesitan recursos institucionales y especialmente políticos que permitan el éxito de esta propuesta. Con todo, existen antecedentes que conviene mencionar, pues sirven de soporte y experiencia al respecto.

Quizás los más representativos de esos antecedentes descansan en los catálogos del Centro Regional de Sismología para América del Sur, CERESIS, que cubren a América del Sur como región. Estos trabajos, ciertamente, tienen como objeto de estudio a los temblores, y no toman en cuenta (salvo en el caso de las manifestaciones eruptivas con efectos sísmicos) a los volcanes ni a otros fenómenos. El CERESIS, con sede en Lima, ha producido diversos trabajos de investigación en el área, incluyendo catalogaciones en países andinos y en la región suramericana. Entre sus publicaciones destacan al respecto el Catálogo Sísmico América del Sur 19921997, que supone un listado de parámetros; ${ }^{40}$ los nueve volúmenes dedicados al Catálogo de Terremotos para América del Sur, conocido como «Catálogos SISRA»;41 y el trabajo de Enrique Silgado. ${ }^{42}$ Asimismo, el CERESIS ha estimulado el estudio histórico de los terremotos en las naciones que más afectadas se ven por estos eventos, y la investigación de Alberto Giesecke y Enrique Silgado, resulta un ejemplo en ese sentido. ${ }^{43}$ Otros catálogos regionales han contribuido con la información histórica de los temblores, como el trabajo del ingeniero venezolano José Grases. ${ }^{44}$

40 CERESIS, 1998.

41 Programa para la Mitigación de los Efectos de los Terremotos en la Región Andina, Proyecto SISRA, coordinado por CERESIS y desarrollado entre 1985 y 1997.

42 Silgado, 1985.

43 Giesecke y Silgado, 1981.

44 Grases, 1990. 
A nivel nacional, los países que más han trabajado en la catalogación han apuntado también sus esfuerzos fundamentalmente hacia la información sísmica. México y Venezuela cuentan con catálogos multimetodológicos y multidisciplinares al respecto que han servido de guía para proyectos internacionales de mayor alcance: Virginia García Acosta y Gerardo Suárez Reynoso, ${ }^{45}$ en el caso de México; mientras que en Venezuela se cuenta con los trabajos de José Grases, Rogelio Altez y Miguel Lugo (1999); ${ }^{46}$ Rogelio Altez y José Antonio Rodríguez; ${ }^{47}$ y el Sistema de Teleinformación de la Sismología Histórica de Venezuela.$^{48}$ Otros países han adelantado estudios y compilaciones en el mismo tema; en Chile: Alfredo Palacios; ${ }^{49}$ en Perú: Lizardo Seiner; ${ }^{50}$ en Colombia: Armando Espinosa Baquero, y la obra clásica de Jesús Emilio Ramírez; ${ }^{51}$ en Ecuador: Manuel Espinosa. ${ }^{52}$

Los volcanes no han tenido el mismo éxito que los terremotos, y de ello solo es posible mencionar un par de trabajos: Armando Espinosa Baquero, ${ }^{53}$ para el caso colombiano; y María Eugenia Petit-Breuilh Sepúlveda, ${ }^{54}$ en una investigación que toma en cuenta a los volcanes de Latinoamérica. De esta última autora se cuenta con otra obra que atiende a los desastres de manera más general, pero con el enfoque territorial ampliado que se pretende destacar aquí y que ha sido referido anteriormente..$^{55}$

Más recientemente, y como esfuerzos institucionales enfocados precisamente en el caso de los sismos, se vienen desarrollando proyectos multinacionales con fines científicos específicos, como lo representa la propuesta del Catálogo de terremotos actualizado y homogéneo para la región Suramericana, elevada ante la iniciativa GEM (Global Earthquake Model) por medio de investigadores de diferentes centros especializados en el área: Istituto Nazionale di Geofisica e Vulcanologia, sección de Milano; Fundación Venezolana de Investigaciones Sismológicas; Instituto Nacional de Prevención Sísmica de Argentina; Observatorio San Calixto, La Paz;

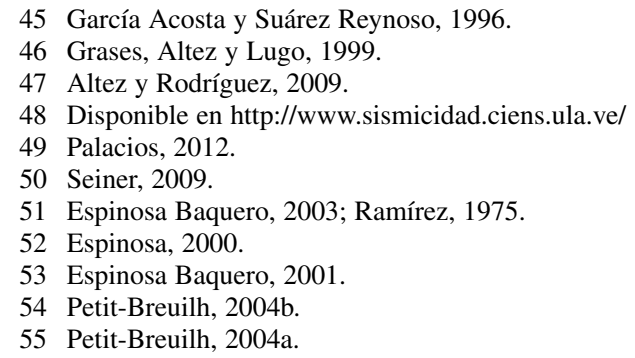


Servicio Geológico Colombiano; Instituto Geofísico de la Escuela Politécnica Nacional, Quito; y otras instancias académicas y universitarias. ${ }^{56}$

Otros fenómenos no han despertado el mismo interés que los temblores y las erupciones, de manera que resulta difícil hallar catálogos de otras manifestaciones regulares de la naturaleza que den cuenta de sus impactos y efectos. Una excepción lo representa el trabajo de Virginia García Acosta, Juan Manuel Pérez Zevallos y América Molina Del Villar, Desastres Agrícolas en México. Catálogo Histórico (2003), que representa un compendio importantísimo de información vinculada a las sequías en ese territorio, fundamentalmente.

El caso de los huracanes resulta emblemático. ${ }^{57}$ Este fenómeno posee temporadas anuales claramente registradas, pero no existe una catalogación precisa sobre sus efectos más allá de investigaciones aisladas o de observatorios modernos fundados para atender el problema a partir de políticas nacionales. Se sabe de eventos que han impactado en zonas tan distantes como la isla de Margarita en Venezuela y el Golfo de México, pero que debido a la ausencia de estudios internacionales al respecto, esos casos hoy aparecen confundidos con lluvias extraordinarias en algunos estudios puntuales. De ellos destaca el trabajo de Raymundo Padilla, el investigador mexicano que ha impulsado el proyecto Los huracanes en la historia de México. Memoria y catálogo, que pronto tendrá productos acabados. ${ }^{58}$

Recuperando la memoria oral en comunidades de la península de Baja California Sur, Padilla indica la utilidad de este recurso para contrastar la información recabada con la información histórica, sosteniendo que «los testimonios de los sobrevivientes y las gráficas de los impactos pueden aportarnos otras luces para caracterizar al fenómeno hidrometeorológico», a partir de la necesidad científica de categorizar los efectos de los huracanes del pasado de los que no se cuenta con información en esa zona. Y destacando la necesidad de contar con herramientas interpretativas adecuadas, nos explica cómo es que en esa recuperación oral puede llegar a confundirse un mismo evento de acuerdo a la terminología característica de los habi-

56 Gómez Capera y Rendón, 2013.

57 Un primer listado al respecto fue elaborado en el Seminario sobre los huracanes del Caribe, que se llevó a cabo en República Dominicana en 1957. Véase, García Bonelly, 1957, 229-234.

58 Véase el blog http://huracanes.ciesas.edu.mx/ sostenido desde el año 2009 con apoyo del CIESAS y el CONACYT, y el trabajo de Padilla, 2006, o la obra colectiva Memoria en movimiento. Testimonios lingüísticos, literarios y visuales sobre el sismo del 21 de 2003 en el estado de Colima, 2003. 
tantes: «Los Choyeros [habitantes de la zona] suelen llamar a los ciclones tropicales como "chubascos, tifones, temporales, ciclones y huracanes" $\gg .{ }^{59}$

Con todo, la mayoría de las iniciativas mencionadas, especialmente las que tienen que ver con los terremotos y los volcanes, apuntan al conocimiento especializado sobre las amenazas naturales que estudian, y no necesariamente representan esfuerzos de investigación que atiendan el problema desde el punto de vista sociológico, antropológico o histórico. Menos aún asumen como un objetivo a la problemática de las fronteras, algo que estiman como un escollo administrativo-institucional, o técnico, en todo caso.

El trabajo que adelanta Raymundo Padilla y la metodología que aplica, al cruzar la información oral y la documental en clave de complementariedad, nos parece el enfoque necesario para acometer la reconstrucción de la memoria colectiva en general, pero específicamente en relación con la problemática que observamos en las regiones fronterizas latinoamericanas y las amenazas compartidas entre dos o más países, reflejada sobre las comunidades asentadas en esas zonas y la multiplicación heterogénea de sus riesgos.

Los problemas (de cualquier índole) que hoy envuelven a la mayoría de esas regiones fronterizas, solo potencian sus vulnerabilidades, e incluso las hacen cada día más vulnerables. Está claro, además, que todos los problemas que padece una sociedad son la expresión cristalizada de procesos históricos que han producido esas características. En ello no solo se advierten los conflictos tradicionalmente identificados con esas zonas y generalmente atendidos como conflictos «compartidos» por los países limítrofes (tráficos ilegales, movimientos poblacionales no controlados, integración, seguridad, ecología, energía, servicios, entre otros), sino también aquellos que eventualmente han sido tratados desde un solo lado de cada borde, como el de los riesgos, las amenazas naturales y las vulnerabilidades. Esta atención parcelada del asunto solo alcanza un trato fragmentario del mismo, como todo lo que sucede en torno a la problemática de los límites nacionales.

Pensamos que las amenazas naturales de mayor potencialidad destructora o de altas probabilidades de producir padecimientos extremos (como los sismos, los volcanes, o las anomalías meteorológicas), representan una destacada oportunidad para iniciar investigaciones enfocadas en la

59 Padilla, 2011. 
reconstrucción de memorias colectivas más articuladas con las realidades fenoménicas, ambientales y climáticas en las que hacen vida las comunidades allí asentadas. Pero esas amenazas también representan una combinación de problemas transfronterizos y transversales, y ante ello parece necesario desplegar una perspectiva que observe a los riesgos como aspectos que deben desnacionalizarse, indefectiblemente. De lo contrario, desde la reproducción de las acciones que tradicionalmente han sido conducidas por estrategias públicas propias de cada país involucrado en esas fronteras, solo continuarán reproduciéndose los riesgos, profundizando las vulnerabilidades, y perdiendo las inversiones en ofertas limitadas y clientelares que apenas rozan los problemas sin hallar solución aparente al asunto.

$\mathrm{Al}$ mismo tiempo, y tomando en cuenta que los desastres son el resultado catastrófico de procesos históricos y sociales, se considera que la reconstrucción histórica de los procesos de desastres contribuiría a la recuperación del pasado de una sociedad, desde una perspectiva analítica y crítica con la cual observar, y sobre todo comprender, la producción y reproducción de las vulnerabilidades y los riesgos como procesos concretamente humanos. Una memoria colectiva que apunte a la consolidación simbólica de la sociedad (y no a su articulación ideológica con los gobiernos de turno), ha de incorporar a sus elementos más característicos aquellos aspectos que le recuerdan al colectivo la existencia de las amenazas con las que convive, independientemente de la calidad y el origen de las mismas. Asirse a una lógica por el estilo tendrá como resultado, por lo menos, el entendimiento acerca del origen histórico y social de los desastres, algo distinto a la común y desacertada calificación de «naturales», noción que responsabiliza a los fenómenos de sus resultados adversos en los contextos humanos. Comprender esto representaría un sólido paso hacia adelante en la reducción de las vulnerabilidades de toda sociedad.

Por encima de cualquier ejemplo, la reconstrucción de las memorias colectivas regionales se levanta como un recurso de defensa y reforzamiento social-simbólico ante la intervención sostenida y permanente de la aplanadora ideológica nacional. En el caso de las regiones fronterizas, desde luego, este reforzamiento estructural podría conducir a la reducción de los riesgos, ya se trate de amenazas naturales, o bien de las peores, las de origen propiamente humano.

La idea de reconstruir las memorias colectivas de la mano de herramientas múltiples y la recuperación de la información histórica con ese destino, pretende hacer de la memoria una herramienta aplicada para, en 
este caso, reducir riesgos asociados con amenazas naturales. La transformación del conocimiento histórico en memoria amplía los horizontes interpretativos de la investigación académica o profesional y persigue, también, competir con los dispositivos institucionales y públicos, siempre anclados en estrategias políticas con fines ideológicos. Una memoria aplicada debería contrarrestar las fuerzas centrípetas de los Estados nacionales y fortalecer subjetiva, simbólica y estructuralmente a las comunidades que tradicionalmente han sido clientes involuntarios de intereses que se hallan muy lejos de valorar su pasado. En cualquier caso, una memoria que alcance a aplicarse en favor de la sociedad, siempre redundará en el fortalecimiento estructural de esa sociedad, donde quiera que se encuentre asentada.

Recibido el 1 de septiembre de 2014 Aceptado el 19 de marzo de 2015

\section{Bibliografía}

Altez, Rogelio: «Historia sin memoria: la cotidiana recurrencia de eventos desastrosos en el estado Vargas, Venezuela», Revista Geográfica Venezolana, Volumen Especial, Mérida, 2005, 313-342.

Altez, Rogelio: Si la naturaleza se opone... Terremotos, historia y sociedad en Venezuela, Caracas, Editorial Alfa, 2010.

Altez, Rogelio: «Independencia, mito genésico y memoria esclerotizada», en Quintero, Inés (coord.), El relato invariable. Independencia, mito y nación, Caracas, Editorial Alfa, 2011, 19-56.

Altez, Rogelio: «1812: Terremotos de perpetua memoria. Aniversarios, conmemoraciones y acuerdos públicos en torno a los sismos del 26 de marzo en Venezuela», Boletín de la Academia Nacional de la Historia, 378, Caracas, 2012, 105-136.

Altez, Rogelio y Rodríguez, José Antonio (coords.): Catálogo Sismológico Venezolano del siglo XX. Documentado e ilustrado, Caracas, Fundación Venezolana de Investigaciones Sismológicas, 2009.

Anderson, Benedict: Comunidades imaginadas, Buenos Aires, Fondo de Cultura Económica, 1993.

Audefroy, Joel F.: «Adaptación de la vivienda vernácula a los climas en México», en García Acosta, Virginia; Audefroy, Joel Francis y Briones, Fernando (coords.), Estrategias sociales de prevención y adaptación, México, Centro de Investigaciones y Estudios Superiores en Antropología Social, 2012, 95-106. 
Augé, Marc: Las formas del olvido, Barcelona, Editorial Gedisa, 1998.

Aurell, Jaume: La escritura de la memoria. De los positivismos a los postmodernismos, Valencia, Publicacions de la Universitat de València, 2005.

Baralt, Rafael María y Díaz, Ramón: Resumen de la Historia de Venezuela [1841], Brujas-París, Desclée de Broker, 1939.

Breuilly, John, Nacionalismo y Estado, Barcelona, Ediciones Pomares-Corredor, 1990.

Briones, Fernando: «Saberes climáticos en la agricultura de los ch'oles de Chiapas», en García Acosta, Virginia; Audefroy, Joel Francis y Briones, Fernando (coords.), Estrategias sociales de prevención y adaptación, México, Centro de Investigaciones y Estudios Superiores en Antropología Social, 2012, 107-112.

Cardozo Galué, Germán: Venezuela: de las regiones históricas a la nación, Caracas, Academia Nacional de la Historia, 2005.

Carrera Damas, Germán: Historia de la historiografía venezolana, Caracas, Ediciones de la Biblioteca Central de la Universidad Central de Venezuela, 1979.

Chust, Manuel y Serrano, José Antonio (eds.): Debate sobre las independencias iberoamericanas, Madrid, AHILA/Iberoamericana/Vervuert, 2007.

Espinosa Baquero, Armando: Erupciones históricas de los volcanes colombianos (1500-1995), Bogotá, Academia Colombiana de Ciencias Exactas, Físicas y Naturales, 2001.

Espinosa Baquero, Armando: Historia Sísmica de Colombia, 1550-1830, Armenia, Academia Colombiana de Ciencias Exactas, Físicas y Naturales/Universidad del Quindío, 2003.

Espinosa, Manuel (comp.): Historia de los Terremotos y las erupciones volcánicas en el Ecuador, siglo XVI-XX, Quito, Taller de Estudios Andinos, 2000.

Ferrer, Carlos y Laffaille, Jaime: «El alud sísmico de La Playa: Causas y efectos del terremoto de Bailadores de 1610», Revista Geográfica Venezolana, 39, 1 y 2, Mérida, 1998, 23-86.

Follari, Roberto: Sobre la desfundamentación epistemológica contemporánea, Caracas, Colección Cátedra de Estudios Avanzados, CIPOST, 1998.

Freud, Sigmund: El malestar en la cultura y otros ensayos, Madrid, Alianza Editorial, 1970.

García Acosta, Virginia: «El estudio histórico de los desastres», en García Acosta, Virginia (coord.), Historia y Desastres en América Latina, Volumen I, Bogotá, LA RED/CIESAS, 1996, 5-22.

García Acosta, Virginia: Los sismos en la historia de México, Tomo 2, México, Universidad Nacional Autónoma de México/Fondo de Cultura Económica, 2001.

García Acosta, Virginia: «La perspectiva histórica en la antropología del riesgo y del desastre. Acercamientos metodológicos», Relaciones. Estudios de Historia y Sociedad, 97, XXV, Zamora (Michoacán), 2004, 125-142. 
García Acosta, Virginia: «El riesgo como construcción social y la construcción social del riesgo», Desacatos. Revista de Antropología Social, 19, México D.F., 2005, 11-24.

García Acosta, Virginia y Suárez Reynoso, Gerardo: Los sismos en la historia de México, Tomo 1, México, Universidad Nacional Autónoma de México/ Fondo de Cultura Económica, 1996.

García Bonelly, Juan Ulises: «Ciclones que han producido daños en la isla Hispaniola», Seminario sobre los huracanes del Caribe, República Dominicana, Talleres Tipográficos Librería Dominicana, 1957, 229-234.

Gellner, Ernest: Naciones y nacionalismos, Madrid, Alianza Editorial, 1988.

Giesecke, Alberto y Silgado, Enrique: Historia de los terremotos en el Perú, Lima, Ediciones Rikchay, 1981.

Gómez Capera, A. Antonio y Rendón, Herbert: Creación de un Catálogo Sísmico Homogéneo para Sur América. Documento de trabajo presentado a la Fundación GEM, 2013. Inédito.

González de Valdés, Gabriela; Sánchez Peña, Ada A.; Delgado Castro, Manuel; Padilla, Raymundo y. Zamorano Manríquez, Luis E: Memoria en movimiento, testimonios lingüísticos, literarios y visuales sobre el sismo del 21 de enero de 2003 en el estado de Colima, México, Universidad de Colima, 2003.

Grases, José: Terremotos Destructores del Caribe, 1502-1990, Montevideo, UNESCO/RELACIS, 1990.

Grases, Jose; Altez, Rogelio y Lugo, Miguel: Catálogo de Sismos Sentidos o Destructores, Venezuela 1530-1998, Caracas, Academia de Ciencias Físicas, Matemáticas y Naturales/Facultad de Ingeniería de la Universidad Central de Venezuela, 1999.

Halbwachs, Maurice: «Memoria colectiva y memoria histórica» (traducción de un fragmento del capítulo II de La mémoire collective, Paris, Les Presses Universitaires de France, 1968), Reis, Revista Española de Investigaciones Sociológicas, 69, Madrid, 1995, 209-219.

Hobsbawm, Eric: Naciones y nacionalismo desde 1780, Barcelona, Crítica/Grijalbo Mondadori, 1991.

Mato, Daniel: «Estudio Introductorio», en Mato, Daniel (coord.), Teoría y política de la construcción de identidades y diferencias en América Latina y el Caribe, Caracas, UNESCO/Editorial Nueva Sociedad, 1994, 13-28.

Moseley, Michael E.: «Modeling Protracted Drought, Collateral Natural Disaster, and Human Responses in the Andes», en Hoffman, Susanna y Oliver-Smith, Anthony, Catastrophe and culture: the anthropology of disaster, Scholl of American Research, James Currey, Santa Fe/Oxford, 2001, 187-212.

Nieto López, Judith: «El deber de la memoria, la imposibilidad del olvido. Alcances ético-políticos», Reflexión Política, 15, 8, Bucaramanga, 2006, 80-92.

Oliver, Paul: Cobijo y sociedad, Madrid, Blume, 1978. 
Padilla, Raymundo: El Huracán del 59, historia del desastre y la reconstrucción de Minatitlán, Universidad de Colima, Secretaría de Cultura del Estado de Colima y Ayuntamiento de Minatitlán, 2006.

Padilla Raymundo: «El "chubasco" del 58 en San José del Cabo, BCS», http://raypadilla.wordpress.com/category/memoria/, publicado el 28 mayo de 2011.

Palacios, Alfredo: Sismicidad histórica de la ciudad de Concepción desde su fundación en 1550 hasta su traslado en 1751, Santiago de Chile, Servicio Nacional de Geología y Minería de Chile, 2012.

Palme, Christl y Altez, Rogelio: «Los sismos de 1673 y 1674 en los Andes venezolanos», Interciencia, 27, 5, Caracas, 2002, 220-226.

Pérez Vejo, Tomás: Elegía criolla. Una interpretación de las guerras de independencia hispanoamericanas, México, Tusquets Editores, 2010.

Petit-Breuilh Sepúlveda, María Eugenia: Desastres naturales y ocupación del territorio en Hispanoamérica, Huelva, Universidad de Huelva, 2004a.

Petit-Breuilh Sepúlveda, María Eugenia: La historia eruptiva de los volcanes hispanoamericanos (siglos XVI al XX), Huelva, Casa de los Volcanes, 2004b.

Petit-Breuilh Sepúlveda, María Eugenia: «Volcanes fronterizos en América Latina: el caso de Chile», Human and Environmental Security in Cross-border Regions: Multidisciplinary approaches in Latin America, LABEL Workshop, Luxembourg, 10-11 October 2013, ponencia.

Quintero, Inés: «Presentación», en Quintero, Inés (coord.), El relato invariable. Independencia, mito y nación, Caracas, Editorial Alfa, 2011, 9-18.

Ramírez, Jesús Emilio: Historia de los terremotos en Colombia, Bogotá, Instituto Geográfico Agustín Codazzi, 1975.

Rapoport, Amos: House, Form and Culture, New Jersey, Englewood Cliffs, 1969.

Revet, Sandrine: Anthropologie d'une catastrophe. Les coulées de boue de 1999 au Venezuela, Paris, Presses Sorbonne Nouvelle, 2007.

Ricoeur, Paul: La memoria, la historia, el olvido, México, Fondo de Cultura Económica, 2004.

Seiner, Lizardo: Historia de los sismos en el Perú. Catálogo: siglos XV-XVII, Lima, Universidad de Lima, 2009.

Silgado, Enrique: Terremotos destructivos en América del Sur, 1530-1984, Lima, CERESIS, 1985.

Vilar, Pierre: Memoria, historia e historiadores, Granada, Universidad de Granada-Universidad de Valencia, 2004. 